\title{
Addendum to "Compact perturbative expressions for neutrino oscillations in matter"
}

\author{
Peter B. Denton ${ }^{a}$ and Stephen J. Parke ${ }^{b}$ \\ ${ }^{a}$ Niels Bohr International Academy, Niels Bohr Institute, University of Copenhagen, \\ Blegdamsvej 17, 2100, Copenhagen, Denmark \\ ${ }^{b}$ Theoretical Physics Department, Fermi National Accelerator Laboratory, \\ P. O. Box 500, Batavia, IL 60510, U.S.A. \\ E-mail: peterbd1@gmail.com, parke@fnal.gov
}

\begin{abstract}
In this Addendum we rewrite the neutrino mixing angles and mass squared differences in matter given in our original paper, [1], in a notation that is more conventional for the reader. Replacing the usual neutrino mixing angles and mass squared differences in the expressions for the vacuum oscillation probabilities with these matter mixing angles and mass squared differences gives an excellent approximation to the oscillation probabilities in matter. Comparisons for T2K \& T2HK, NOvA, T2HKK and DUNE are also given for neutrinos and anti-neutrinos, disappearance and appearance channels, and for both normal and inverted orderings.
\end{abstract}

Keywords: CP violation, Beyond Standard Model, Neutrino Physics

ArXIV EPRINT: 1801.06514 


\section{Contents}

1 Introduction 1

2 Mixing angles and mass differences in matter 2

2.1 Zeroth order 2

2.2 Higher orders 4

3 Oscillation probabilities $\quad 6$

$\begin{array}{ll}3.1 \text { Comparisons } & 6\end{array}$

$\begin{array}{lll}4 & \text { Summary } & 7\end{array}$

$\begin{array}{ll}\text { A Asymptotic expansions } & 16\end{array}$

$\begin{array}{ll}\text { B Comparison between this addendum and DMP } & 16\end{array}$

\section{Introduction}

Current and next generation long baseline accelerator neutrino experiments will measure at high significance the remaining unknowns of neutrino oscillations. These experiments take advantage of the fact that neutrino oscillations are modified by the presence of matter. In [1] (DMP) we presented a new method of describing oscillation probabilities in matter for all channels, energies, and baselines that is simple and precise. The method relies on changing the basis by modifying two of the mixing angles, $\theta_{13}$ and $\theta_{12}$, to their matter values to remove level crossings, followed by a perturbative expansion.

In this Addendum we rewrite the expressions given in our original paper, [1], in a notation that is more conventional and commonly used to further simplify our expressions. It is then straightforward to replace the usual neutrino mixing angles and mass squared differences in the expressions for the vacuum oscillation probabilities with these simple modified matter mixing angles and mass squared differences which in turn give an excellent approximation to the oscillation probabilities in matter. We also show that higher orders are also easily calculated and the precision improves by several orders of magnitude per order.

In section 2, we give the approximation to the mixing angles and mass squared difference in matter and discuss how to use these to calculated the oscillation probabilities in matter both at 0th order and 1st order. We also give expansions of the mixing angles and mass squared differences in matter in powers of $\left(a / \Delta m^{2}\right)$ in appendix A. In section 3, we make a detailed comparison between the exact and the approximate oscillation probabilities in matter for the T2K \& T2HK $(295 \mathrm{~km})$, NOvA $(810 \mathrm{~km})$, T2HKK $(1050 \mathrm{~km})$ and DUNE $(1300 \mathrm{~km})$ experiments. Section 4 is the Summary. 


\section{Mixing angles and mass differences in matter}

Once the mixing angles mass differences in matter are expressed, the oscillation probabilities follow directly by taking the vacuum expressions and replacing the vacuum parameters with those in matter derived below. Improvements beyond zeroth order can be achieved by perturbative expansions by modifying the oscillation probabilities beyond the vacuum expressions.

\subsection{Zeroth order}

An alternative way to write the matter mixing angles, $\theta$ 's, and $\Delta m^{2}$ 's in matter, derived in DMP, is given in this section. We perform two successive two-flavor rotations which are carefully and precisely chosen to maximize the accuracy of the zeroth order approximation. For these two-flavor rotations, the mixing angles in matter, $\widetilde{\theta}$, are in range $[0, \pi / 2]$, so $\cos 2 \widetilde{\theta}$ determines them uniquely.

The first rotation is the 1-3 sector, which gives us $\theta_{13}$ in matter which we denoted by $\widetilde{\theta}_{13}$ :

$$
\cos 2 \widetilde{\theta}_{13}=\frac{\left(\cos 2 \theta_{13}-a / \Delta m_{e e}^{2}\right)}{\sqrt{\left(\cos 2 \theta_{13}-a / \Delta m_{e e}^{2}\right)^{2}+\sin ^{2} 2 \theta_{13}}},
$$

where $a \equiv 2 \sqrt{2} G_{F} N_{e} E_{\nu}$ is the standard Wolfenstein matter potential. In this rotation, it is important that $\Delta m_{e e}^{2} \equiv \cos ^{2} \theta_{12} \Delta m_{31}^{2}+\sin ^{2} \theta_{12} \Delta m_{32}^{2}$, from [2, 3], is used instead of either $\Delta m_{31}^{2}$ or $\Delta m_{32}^{2}$, as this seemingly minor changes improves the accuracy of the zeroth order approximation by including some of the first order corrections. The associated two-flavor $\Delta m^{2}$ for this rotation, which simplifies many following expressions, is given by

$$
\Delta \widetilde{m^{2}} e=\Delta m_{e e}^{2} \sqrt{\left(\cos 2 \theta_{13}-a / \Delta m_{e e}^{2}\right)^{2}+\sin ^{2} 2 \theta_{13}} .
$$

We use the symbol $\widetilde{\Delta m^{2}} e e$ here, as this variable is equal to $\Delta m_{e e}^{2}$ in vacuum, but more details after eq. (2.4). by $\widetilde{\theta}_{12}$ :

The second rotation is the 1-2 sector, which gives us $\theta_{12}$ in matter which we denoted

$$
\cos 2 \widetilde{\theta}_{12}=\frac{\left(\cos 2 \theta_{12}-a^{\prime} / \Delta m_{21}^{2}\right)}{\sqrt{\left(\cos 2 \theta_{12}-a^{\prime} / \Delta m_{21}^{2}\right)^{2}+\sin ^{2} 2 \theta_{12} \cos ^{2}\left(\widetilde{\theta}_{13}-\theta_{13}\right)}},
$$

where $a^{\prime}$ is the $\theta_{13}$-modified matter potential for the 1-2 sector and is given by

$$
a^{\prime}=a \cos ^{2} \widetilde{\theta}_{13}+\Delta m_{e e}^{2} \sin ^{2}\left(\widetilde{\theta}_{13}-\theta_{13}\right)=\left(a-\Delta \widetilde{m^{2}} e e+\Delta m_{e e}^{2}\right) / 2,
$$

and $\cos ^{2}\left(\widetilde{\theta}_{13}-\theta_{13}\right)$ can also be written in terms of $\widetilde{\Delta m^{2}}$ ee as follows

$$
\cos ^{2}\left(\widetilde{\theta}_{13}-\theta_{13}\right)=\left(\widetilde{m^{2}} e e+\Delta m_{e e}^{2}-a \cos 2 \theta_{13}\right) /\left(2 \Delta \widetilde{m^{2}} e\right) .
$$

The associated two-flavor $\Delta m^{2}$ for this rotation, $\Delta \widetilde{m^{2}} 21$, is just $\Delta m_{21}^{2}$ in matter, and it is given by

$$
\Delta \widetilde{m^{2}} 21=\Delta m_{21}^{2} \sqrt{\left(\cos 2 \theta_{12}-a^{\prime} / \Delta m_{21}^{2}\right)^{2}+\sin ^{2} 2 \theta_{12} \cos ^{2}\left(\widetilde{\theta}_{13}-\theta_{13}\right)} .
$$


The other $\Delta m^{2}$ 's in matter can be calculated as follows

$$
\begin{aligned}
& \Delta{\widetilde{m^{2}}}_{31}=\Delta m_{31}^{2}+\frac{1}{4} a+\frac{3}{4}\left({\widetilde{m^{2}}}_{e e}-\Delta m_{e e}^{2}\right)+\frac{1}{2}\left(\Delta \widetilde{m^{2}}{ }_{21}-\Delta m_{21}^{2}\right), \\
& \Delta{\widetilde{m^{2}}}_{32}=\Delta{\widetilde{m^{2}}}_{31}-\Delta \widetilde{m^{2}}{ }_{21} .
\end{aligned}
$$

The individual neutrino mass squareds in matter, up to an arbitrary common offset, are

$$
\begin{aligned}
& \widetilde{m_{3}^{2}}=\Delta m_{31}^{2}+\left(a-a^{\prime}\right)=\Delta m_{31}^{2}+\frac{1}{2} a+\frac{1}{2}\left({\widetilde{m^{2}}}_{e e}-\Delta m_{e e}^{2}\right) \\
& \widetilde{m_{2}^{2}}=\frac{1}{2}\left(a^{\prime}+\Delta \widetilde{m^{2}}{ }_{21}+\Delta m_{21}^{2}\right)=\Delta m_{21}^{2}+\frac{1}{4} a-\frac{1}{4}\left(\Delta \widetilde{m^{2}} e e-\Delta m_{e e}^{2}\right)+\frac{1}{2}\left(\Delta \widetilde{m^{2}}{ }_{21}-\Delta m_{21}^{2}\right), \\
& \widetilde{m_{1}^{2}}=\frac{1}{2}\left(a^{\prime}-\Delta \widetilde{m^{2}}{ }_{21}+\Delta m_{21}^{2}\right)=\frac{1}{4} a-\frac{1}{4}\left(\Delta{\widetilde{m^{2}}}_{e e}-\Delta m_{e e}^{2}\right)-\frac{1}{2}\left(\Delta{\widetilde{m^{2}}}_{21}-\Delta m_{21}^{2}\right) .
\end{aligned}
$$

Also, one can show that $\Delta \widetilde{m^{2}} e e \equiv \cos ^{2} \widetilde{\theta}_{12} \Delta \widetilde{m^{2}}{ }_{31}+\sin ^{2} \widetilde{\theta}_{12} \Delta \widetilde{m^{2}} 32$, i.e. the matter generalization of $\Delta m_{e e}^{2}$, explaining why we use the symbol $\widetilde{\Delta m^{2} e e}$ for this variable. The physical meaning of $\Delta m^{2} e$ is that it is $\Delta m_{e e}^{2}$ in matter and together with $\sin ^{2} \widetilde{\theta}_{13}$ dominates $\nu_{e} \rightarrow \nu_{e}$ oscillations in matter, at atmospheric L/E scale, as seen in section 3.3 .5 of [4] and section 3 of [5].

$\theta_{23}$ and $\delta$ remain unchanged in matter for this approximation.

In figure 1 and figure 2 we show the values of matter potentials, a, $\mathrm{a}^{\prime}$, sine squared of the matter mixing angles, $\sin ^{2} \widetilde{\theta}_{13}$ and $\sin ^{2} \widetilde{\theta}_{12}$, the matter mass squared eigenvalues, $\widetilde{m^{2}}{ }_{j}$, and the matter mass squared differences, ${ }^{1} \Delta \widetilde{m^{2}}{ }_{j k}$, as a function of the neutrino energy for a density of 3.0 g.cm ${ }^{-3}$.

To calculate the oscillation probabilities, to 0th order, use the above $\Delta \widetilde{m^{2}}{ }_{j k}$ instead of $\Delta m_{j k}^{2}$ and replace the vacuum PMNS matrix as follows

$$
U_{P M N S}^{0} \equiv U_{23}\left(\theta_{23}\right) U_{13}\left(\theta_{13}, \delta\right) U_{12}\left(\theta_{12}\right) \Rightarrow U_{P M N S}^{M} \equiv U_{23}\left(\theta_{23}\right) U_{13}\left(\widetilde{\theta}_{13}, \delta\right) U_{12}\left(\widetilde{\theta}_{12}\right) .
$$

That is, replace

$$
\begin{array}{rlrl}
\theta_{13} & \rightarrow \widetilde{\theta}_{13} & & \text { given in eq. (2.1), } \\
\theta_{12} & \rightarrow \widetilde{\theta}_{12} & \text { given in eq. (2.2), } \\
\Delta m_{21}^{2} & \rightarrow \Delta \widetilde{m^{2}} 21 & \text { given in eq. (2.3), } \\
\& \quad \Delta m_{31}^{2} & \rightarrow \Delta \widetilde{m^{2}} 31 & \text { given in eq. (2.4), }
\end{array}
$$

and leave $\theta_{23}$ and $\delta$ unchanged; it is that simple. We call this the 0th order DMP approximation. For the connection with the notation in DMP, see appendix B.

These expressions are valid for both NO, $\Delta m_{e e}^{2}>0$ and IO, $\Delta m_{e e}^{2}<0$. For antineutrinos, just change the sign of $a$ and $\delta$. Our expansion parameter is

$$
\left|\sin \left(\tilde{\theta}_{13}-\theta_{13}\right) \sin \theta_{12} \cos \theta_{12} \frac{\Delta m_{21}^{2}}{\Delta m_{e e}^{2}}\right| \leq 0.015,
$$

\footnotetext{
${ }^{1}$ For NO, we plot $(j k)=(31),(32) \&(21)$, whereas for IO we plot $(j k)=(13),(23) \&(21)$, this way all $\Delta m^{2}$ 's plotted are positive.
} 

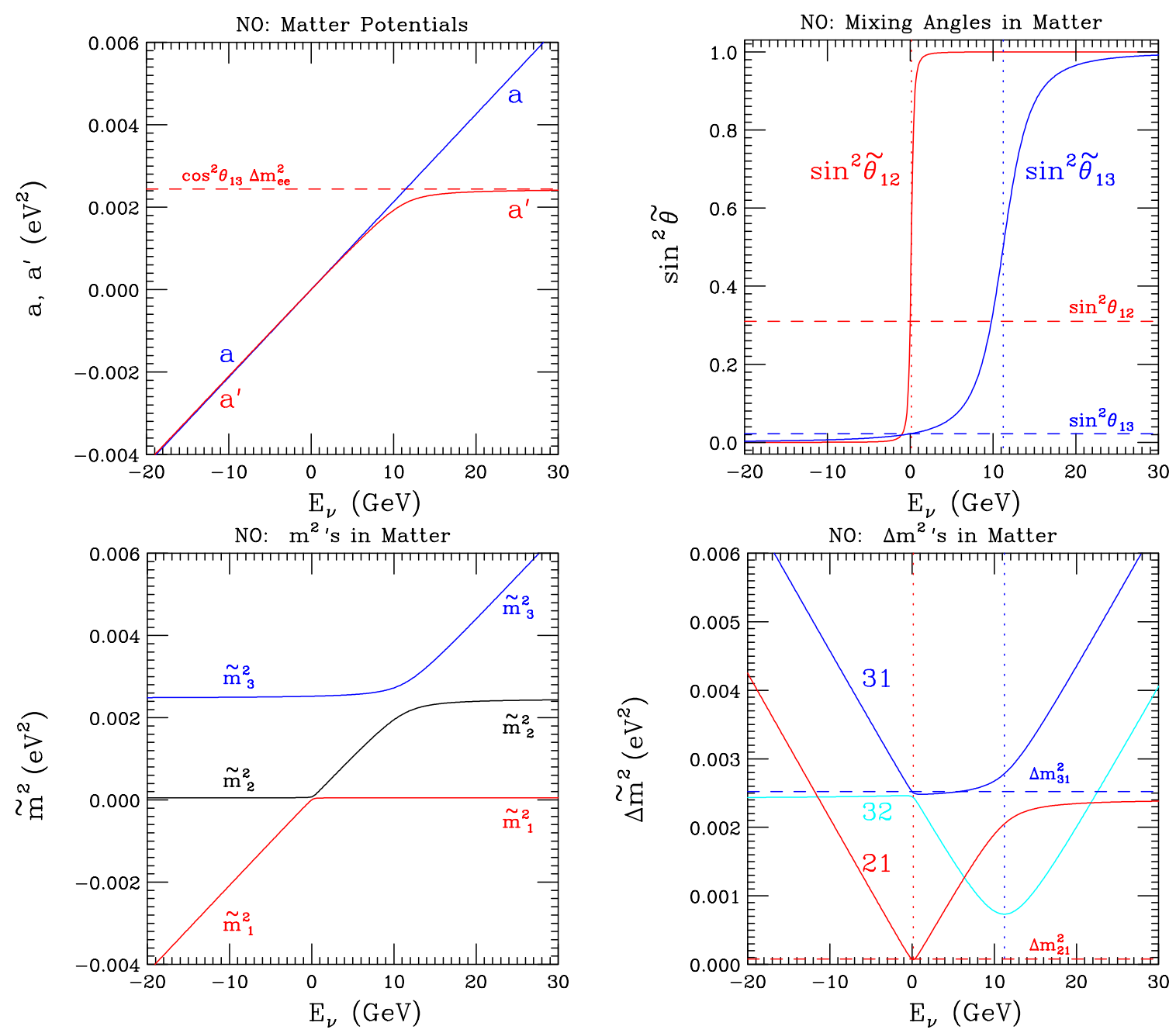

Figure 1. In the normal ordering (NO): top left, the matter potentials, $a$ and $a^{\prime}$, top right, sine squared of mixing angles in matter, $\sin ^{2} \widetilde{\theta}_{j k}$, bottom left, the mass squared eigenvalues in matter, $\widetilde{m^{2}}{ }_{j}$, and bottom right, the mass squared differences in matter, $\Delta \widetilde{m^{2}}{ }_{j k} . E_{\nu} \geq 0\left(E_{\nu} \leq 0\right)$ is for neutrinos (anti-neutrinos). $E_{\nu}=0$ is the vacuum values for both neutrinos and anti-neutrinos.

which is small and vanishes in vacuum, so that our perturbation theory reproduces the vacuum oscillation probabilities exactly.

If $P_{\nu_{\alpha} \rightarrow \nu_{\beta}}\left(\Delta m_{31}^{2}, \Delta m_{21}^{2}, \theta_{13}, \theta_{12}, \theta_{23}, \delta\right)$ is the oscillation probability in vacuum then $P_{\nu_{\alpha} \rightarrow \nu_{\beta}}\left(\Delta \widetilde{m^{2}}{ }_{31}, \Delta \widetilde{m^{2}}{ }_{21}, \widetilde{\theta}_{13}, \widetilde{\theta}_{12}, \theta_{23}, \delta\right)$ is the oscillation probability in matter, i.e. use the same function but replace the mass squared differences and mixing angles with the matter values given in eqs. (2.1)-(2.4). The resulting oscillation probabilities are identical to the zeroth order approximation given in DMP.

\section{$2.2 \quad$ Higher orders}

If the 0th order is not accurate enough, going to 1st order is simple and gives another two orders of magnitude in accuracy. First the $\Delta \widetilde{m^{2}}{ }_{j k}$ remain unchanged but the mixing 

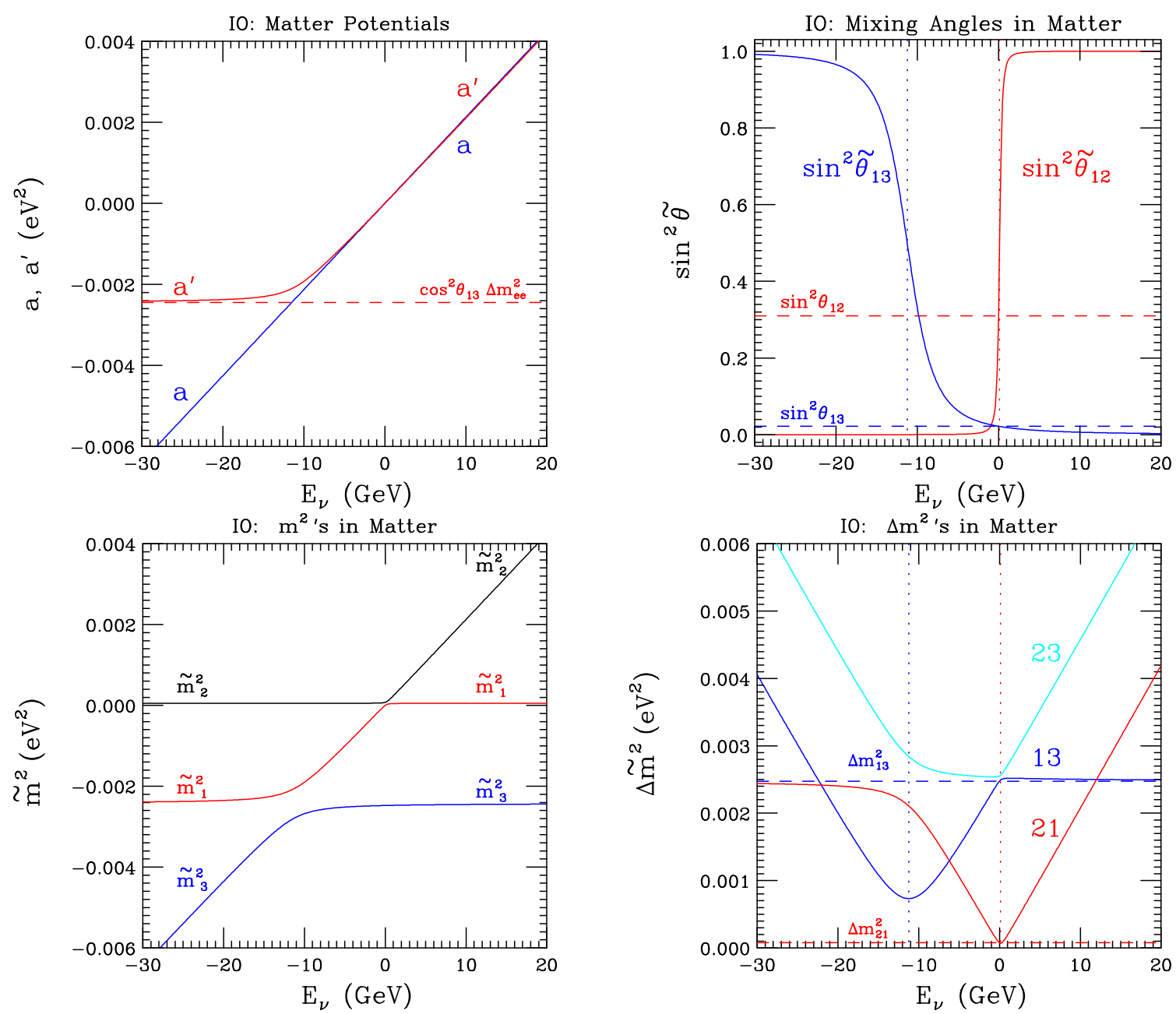

Figure 2. In the inverted ordering (IO): top left, the matter potentials, $a$ and $a^{\prime}$, top right, sine squared of mixing angles in matter, $\sin ^{2} \widetilde{\theta}_{j k}$, bottom left, the mass squared eigenvalues in matter, $\widetilde{m^{2}}{ }_{j}$, and bottom right, the mass squared differences in matter, $\Delta \widetilde{m^{2}}{ }_{j k} . E_{\nu} \geq 0\left(E_{\nu} \leq 0\right)$ is for neutrinos (anti-neutrinos). $E_{\nu}=0$ is the vacuum values for both neutrinos and anti-neutrinos.

matrix is modified by

$$
U_{P M N S}^{M} \Rightarrow V \equiv U_{P M N S}^{M}\left(1+W_{1}\right),
$$

where the matrix $W_{1}$ is given by

$$
\begin{aligned}
& W_{1}=\sin \left(\widetilde{\theta}_{13}-\theta_{13}\right) s_{12} c_{12} \Delta m_{21}^{2} \\
& \left(\begin{array}{ccc}
0 & 0 & -\widetilde{s}_{12} e^{-i \delta} / \Delta{\widetilde{m^{2}}}_{31} \\
0 & 0 & +\widetilde{c}_{12} e^{-i \delta} / \Delta \widetilde{m^{2}} 32 \\
+\widetilde{s}_{12} e^{+i \delta} / \Delta{\widetilde{m^{2}}}_{31} & -\widetilde{c}_{12} e^{+i \delta} / \Delta{\widetilde{m^{2}}}_{32} & 0
\end{array}\right)
\end{aligned}
$$

where $\widetilde{s}_{12}=\sin \widetilde{\theta}_{12}$ and $\widetilde{c}_{12}=\cos \widetilde{\theta}_{12}$ etc. The $\Delta \widetilde{m^{2}}{ }_{j k}$ and the $V$-mixing matrix can be used to calculate the oscillation probabilities and improves the accuracy by two orders of 
magnitude. We call this the 1st order DMP approximation. The next highest order, 2nd order, is also discussed in DMP.

\section{Oscillation probabilities}

\subsection{Comparisons}

The neutrino parameters in vacuum relevant for oscillations are:

$$
\begin{array}{rlrl}
\Delta m_{32}^{2} & = \pm 2.5 \times 10^{-3} \mathrm{eV}^{2}, \quad \Delta m_{21}^{2} & =+7.5 \times 10^{-5} \mathrm{eV}^{2} \\
\sin ^{2} \theta_{12} & =0.31, & \sin ^{2} \theta_{23} & =0.43 \\
\sin ^{2} \theta_{13} & =0.022, & |\delta| & =72^{\circ}=2 \pi / 5,
\end{array}
$$

where $\Delta m_{32}^{2}>0$ gives a normal ordering (NO) neutrino spectrum and $\Delta m_{32}^{2}<0$ gives the inverted ordering (IO). For neutrinos, we use $\delta=-72^{\circ}=-2 \pi / 5$, whereas for antineutrinos, we use $\delta=+72^{\circ}=+2 \pi / 5$, so as to emphasis the matter effect differences between the neutrino and anti-neutrino channels. Note we have avoided the special points: $\theta_{23}=\pi / 4$ as well as $\delta=0, \pm \pi / 2, \pi$, so as not to overestimate the precision.

To be comprehensive we consider four experimental setups with energy windows wider than those accessible for the particular experiment.

- T2K [6]/T2HK [7]: with baseline, $\mathrm{L}=295 \mathrm{~km}$, neutrino energy $0.2<E_{\nu} / \mathrm{GeV}<3.0$, and density, $\rho=2.3$ g.cm ${ }^{-3}$. See figures 3,4 .

- NOvA [8]: with baseline, $\mathrm{L}=810 \mathrm{~km}$, neutrino energy $0.6<E_{\nu} / \mathrm{GeV}<4.0$, and density, $\rho=3.0$ g.cm ${ }^{-3}$. See figures 5,6 .

- T2HKK [9]: with baseline, $\mathrm{L}=1050 \mathrm{~km}$, neutrino energy $0.3<E_{\nu} / \mathrm{GeV}<5.0$, and density, $\rho=3.0$ g.cm ${ }^{-3}$. See figures 7,8 .

- DUNE [10]: with baseline, $\mathrm{L}=1300 \mathrm{~km}$, neutrino energy $0.5<E_{\nu} / \mathrm{GeV}<7.0$, and density, $\rho=3.0$ g.cm ${ }^{-3}$. See figures 9,10 .

In these figures we have considered the channels $\nu_{\mu}$ disappearance and $\nu_{e}$ appearance for neutrinos and anti-neutrinos and for both NO and IO. Each figure consists of three panels: the top panel is the exact oscillation probabilities in matter from Zaglauer and Schwarzer, [11], the 0th order DMP approximation as well as the exact vacuum oscillation probability. In the disappearance channel the difference between the three probabilities is less than the thickness of the lines. In the appearance channel, the difference between exact and 0th order DMP probabilities is less than the thickness of the lines. The middle (bottom) panel shows the difference (fractional difference) between

1. the exact, [11], and vacuum oscillation probabilities (black dots),

2. the exact and the 0th order DMP approximation (solid red),

3. the exact and the 1st order DMP approximation (solid magenta). 


\begin{tabular}{|l|c|c|c|c|}
\hline & T2K/HK & NOvA & T2HKK & DUNE \\
\hline $\max \Delta P$ & $10^{-5}$ & $10^{-4}$ & $10^{-4}$ & $10^{-4}$ \\
$\max \Delta P / P$ & $10^{-3}$ & $10^{-3}$ & $10^{-2.5}$ & $10^{-2}$ \\
\hline
\end{tabular}

Table 1. The maximum $\Delta P$ and $\Delta P / P$ at 0 th order in the DMP approximation. The largest fraction difference occurs at oscillation maximum for $\nu_{\mu}$ disappearance channel, where the oscillation probability is a few \%. None of the experiments included here, T2K \& T2HK, NOvA, T2HKK and DUNE will be within an order a magnitude of being sensitive to any of these differences.

In the fractional differences, the denominator is the average of the two probabilities being compared. In figure 3 to figure 10, the "dips" in middle and bottom panels appear when $\Delta P$ changes sign, see also $[12,13]$.

In table 1 , we give the maximum difference and fractional difference of the 0th order approximation to the exact probability.

\section{Summary}

In DMP, [1], we derived a new perturbative expansion for neutrino oscillation parameters in uniform matter, using as the primary variables, the neutrino mass eigenvalues in matter, which contain all the information about $\Delta m^{2}$ and the mixing angles in matter. In this Addendum we have converted the results of that paper into the more traditional mixing angles and $\Delta m^{2}$ 's in matter which may be more familiar to some readers. This clearer formulation shows how the DMP formulation is based on two two-flavor matter rotations, precisely chosen to give highly accurate results at zero order in a fashion that matches the PMNS matrix. This means that the zeroth order matter expressions take exactly the same functional form as the vacuum expressions. Numerically the neutrino oscillation probabilities using the formulation of this Addendum and that of DMP are identical since they can be shown to be identical analytically.

We have also compared the results of the DMP approximation at 0th and 1st order (it doesn't matter which formulation since they are identical), to the exact results and have shown that DMP at zeroth order is sufficiently accurate for all of the accelerator based neutrino oscillation experiments operating or planned: T2K \& T2HK, NOvA, T2HKK, and DUNE. The first order perturbative expansion, which is also simple to implement, improves the accuracy by a further two orders of magnitude.

\section{Acknowledgments}

This manuscript has been authored by Fermi Research Alliance, LLC under Contract No. DE-AC02-07CH11359 with the U.S. Department of Energy, Office of Science, Office of High Energy Physics.

This project has received funding/support from the European Union's Horizon 2020 research and innovation programme under the Marie Sklodowska-Curie grant agreement No 690575 and No 674896. 


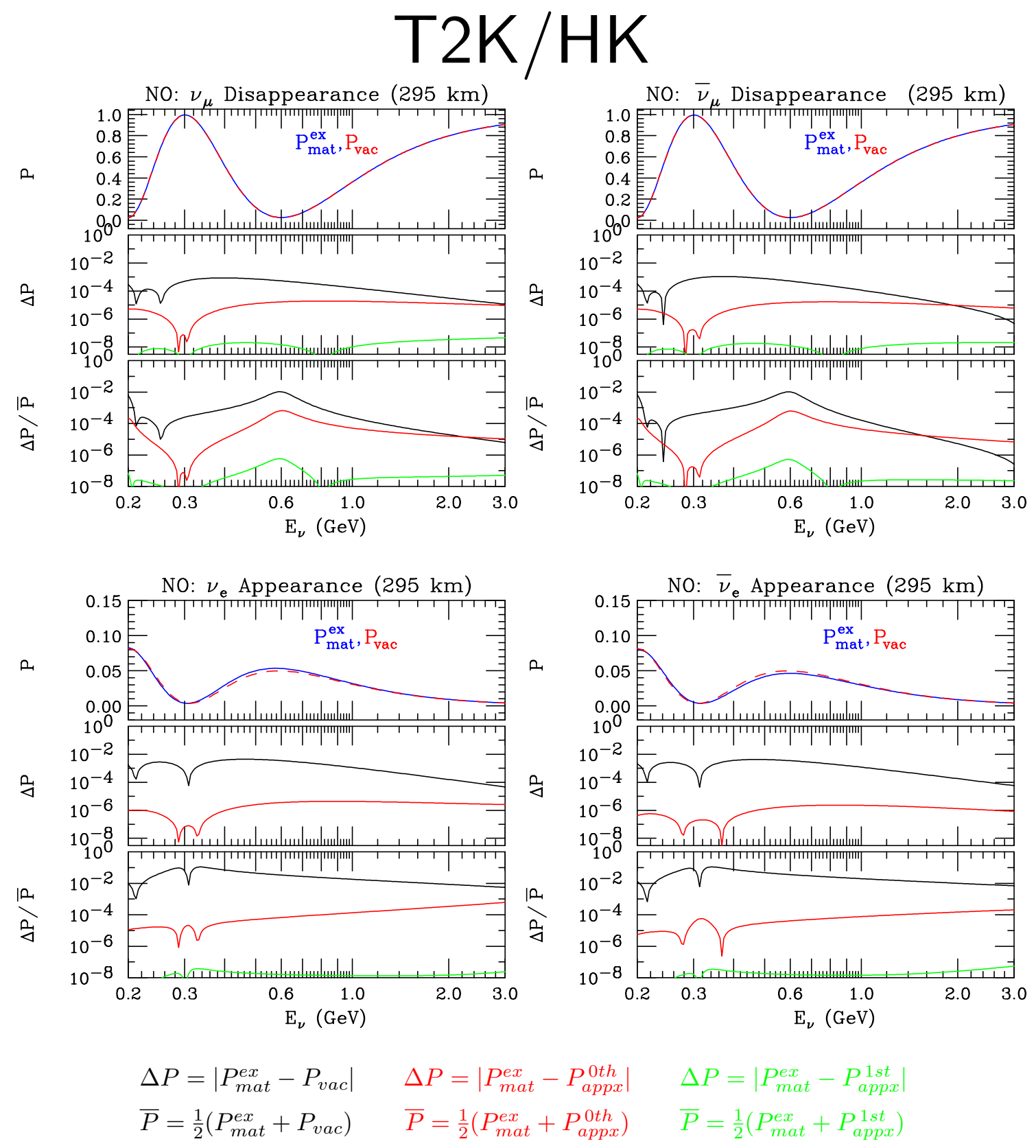

Figure 3. T2K/HK, for normal ordering (NO): top left figure is $\nu_{\mu}$ disappearance, top right figure is $\bar{\nu}_{\mu}$ disappearance, bottom left figure is $\nu_{\mu} \rightarrow \nu_{e}$ appearance, and bottom right is $\bar{\nu}_{\mu} \rightarrow \bar{\nu}_{e}$ appearance. In each figure, the top panel is exact oscillation probability in matter, $P_{m a t}^{e x}$ (blue dashes) from [11], the zeroth order DMP approximation, $P_{a p p x}^{0 \text { th }}$ (red dashes) from [1] and the vacuum oscillation probability, $P_{\text {vac }}$ (black dots). The Middle panel is difference between exact oscillation probabilities in matter and vacuum (black dots), and the difference between exact and 0th DMP approximation (solid red) and exact and 1st DMP approximation (solid magenta) approximations. Bottom panel is similar to middle panel but plotting the fractional differences, $\Delta P / \bar{P}$. The density used is 2.3 g.cm ${ }^{-3}$. 


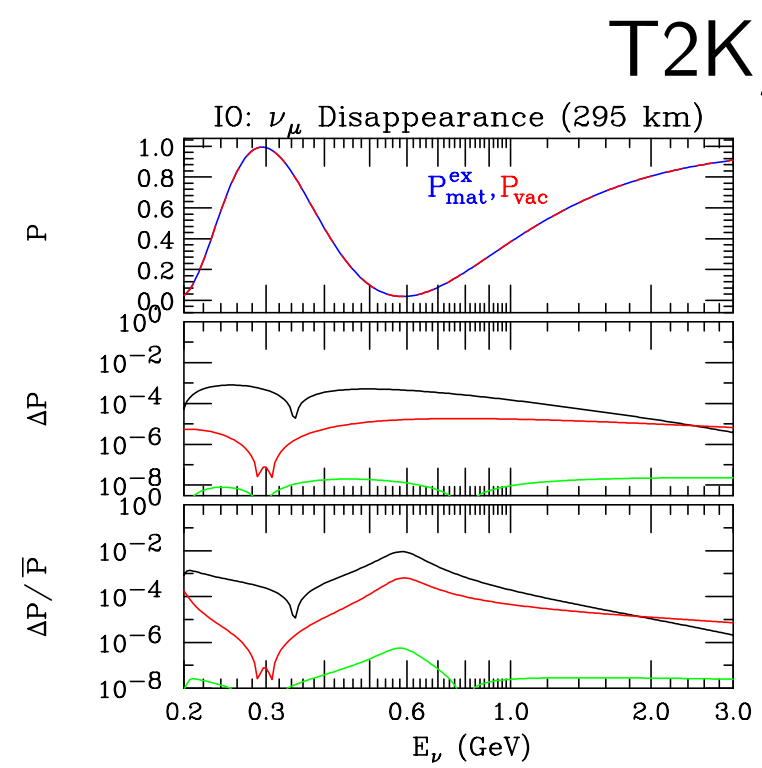

HK
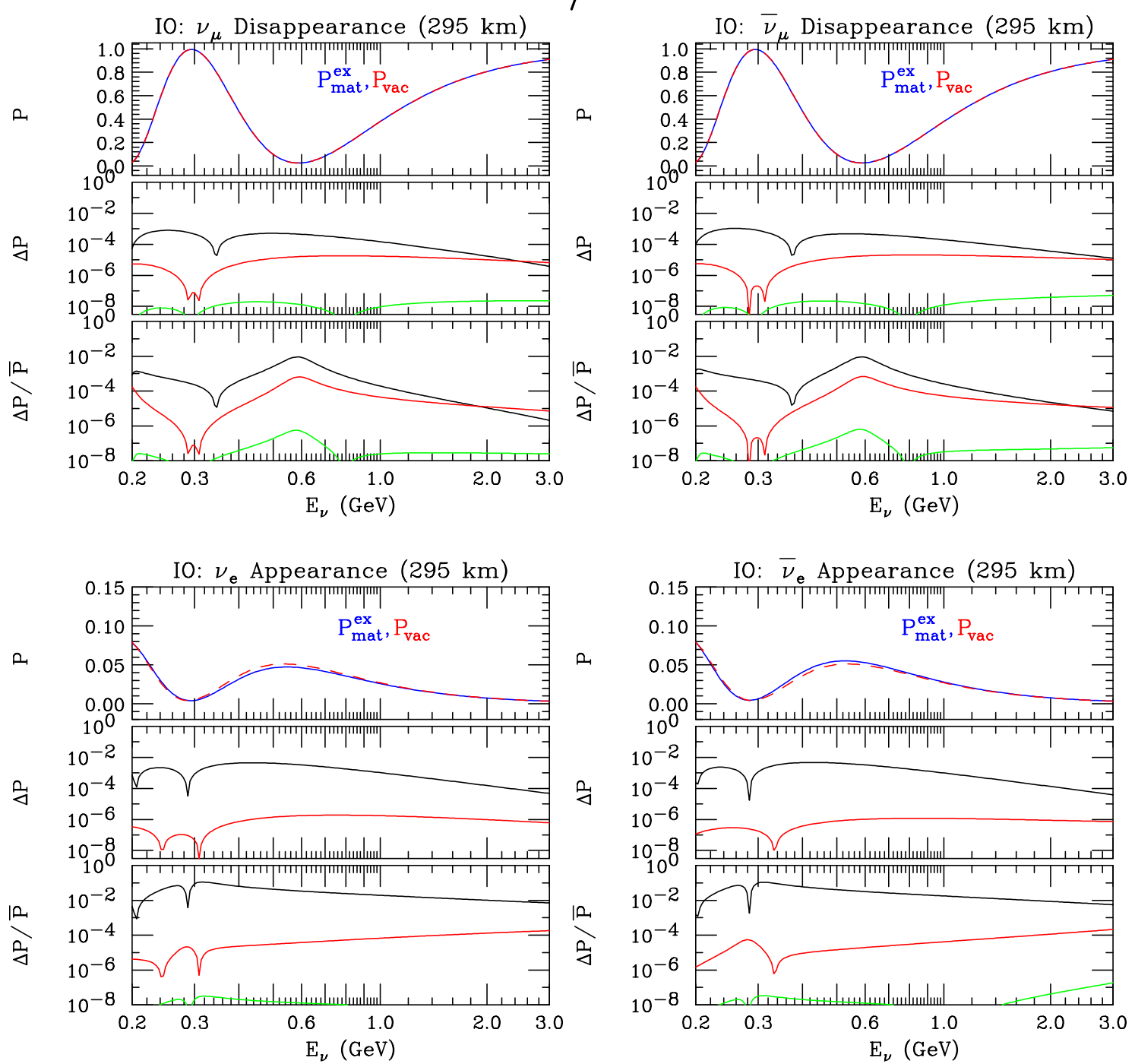

$$
\begin{array}{lll}
\Delta P=\left|P_{m a t}^{e x}-P_{v a c}\right| & \Delta P=\left|P_{m a t}^{e x}-P_{a p p x}^{0 t h}\right| & \Delta P=\left|P_{m a t}^{e x}-P_{a p p x}^{1 s t}\right| \\
\bar{P}=\frac{1}{2}\left(P_{m a t}^{e x}+P_{v a c}\right) & \bar{P}=\frac{1}{2}\left(P_{\text {mat }}^{e x}+P_{a p p x}^{0 t h}\right) & \bar{P}=\frac{1}{2}\left(P_{\text {mat }}^{e x}+P_{a p p x}^{1 s t}\right)
\end{array}
$$

Figure 4. T2K/HK, for inverted ordering (IO): top left figure is $\nu_{\mu}$ disappearance, top right figure is $\bar{\nu}_{\mu}$ disappearance, bottom left figure is $\nu_{\mu} \rightarrow \nu_{e}$ appearance, and bottom right is $\bar{\nu}_{\mu} \rightarrow \bar{\nu}_{e}$ appearance. In each figure, the top panel is exact oscillation probability in matter, $P_{m a t}^{e x}$ (blue dashes) from [11], the zeroth order DMP approximation, $P_{a p p x}^{0 \text { th }}$ (red dashes) from [1] and the vacuum oscillation probability, $P_{v a c}$ (black dots). The Middle panel is difference between exact oscillation probabilities in matter and vacuum (black dots), and the difference between exact and 0th DMP approximation (solid red) and exact and 1st DMP approximation (solid magenta) approximations. Bottom panel is similar to middle panel but plotting the fractional differences, $\Delta P / \bar{P}$. The density used is $2.3 \mathrm{~g} . \mathrm{cm}^{-3}$. 

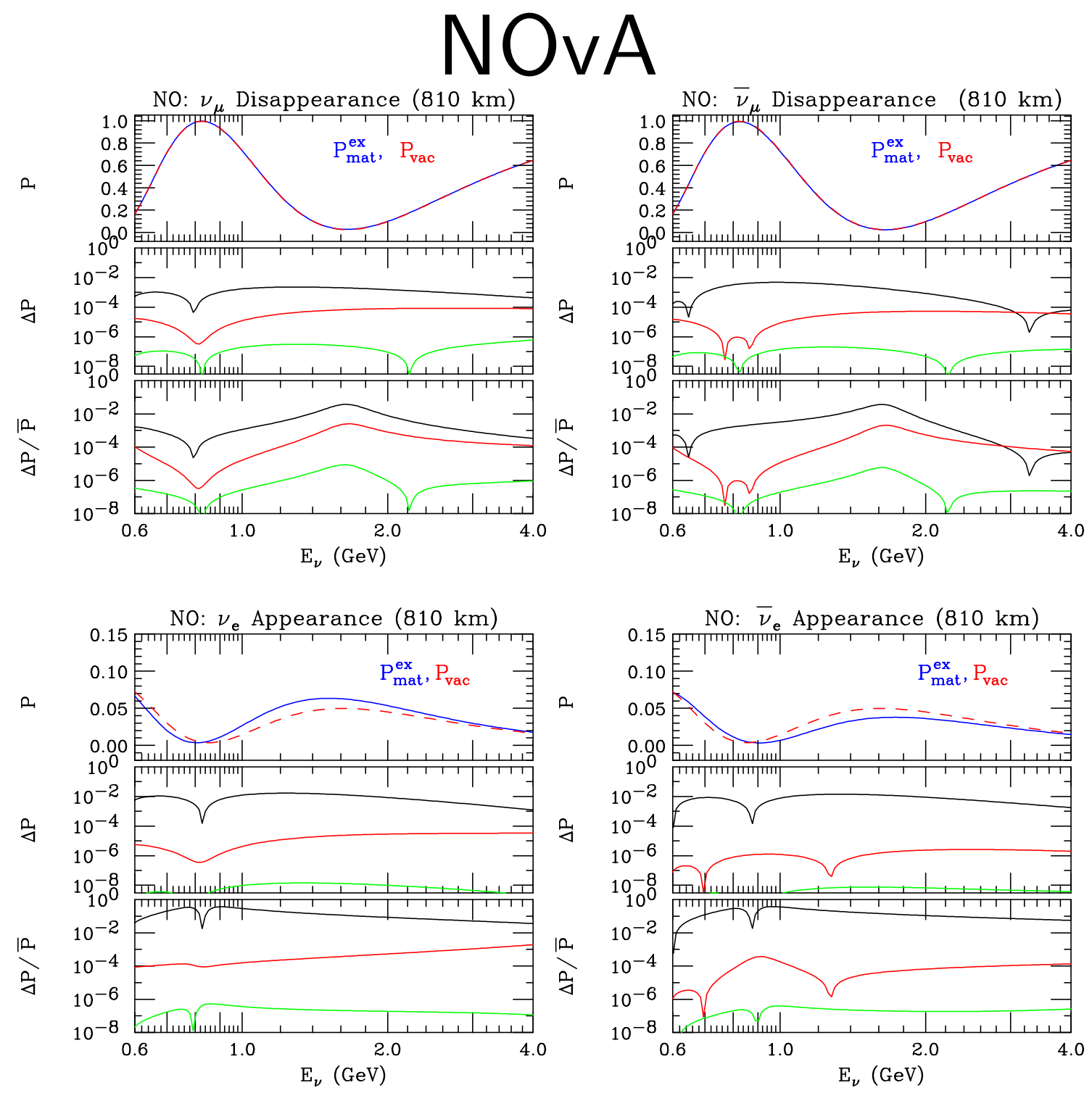

$$
\begin{array}{lll}
\Delta P=\left|P_{m a t}^{e x}-P_{v a c}\right| & \Delta P=\left|P_{m a t}^{e x}-P_{a p p x}^{0 t h}\right| & \Delta P=\left|P_{m a t}^{e x}-P_{a p p x}^{1 s t}\right| \\
\bar{P}=\frac{1}{2}\left(P_{\text {mat }}^{e x}+P_{v a c}\right) & \bar{P}=\frac{1}{2}\left(P_{\text {mat }}^{e x}+P_{a p p x}^{0 t h}\right) & \bar{P}=\frac{1}{2}\left(P_{\text {mat }}^{e x}+P_{\text {app }}^{1 s t}\right)
\end{array}
$$

Figure 5. NOvA, for normal ordering (NO): top left figure is $\nu_{\mu}$ disappearance, top right figure is $\bar{\nu}_{\mu}$ disappearance, bottom left figure is $\nu_{\mu} \rightarrow \nu_{e}$ appearance, and bottom right is $\bar{\nu}_{\mu} \rightarrow \bar{\nu}_{e}$ appearance. In each figure, the top panel is exact oscillation probability in matter, $P_{m a t}^{e x}$ (blue dashes) from [11], the zeroth order DMP approximation, $P_{a p p x}^{0 \text { th }}$ (red dashes) from [1] and the vacuum oscillation probability, $P_{v a c}$ (black dots). The Middle panel is difference between exact oscillation probabilities in matter and vacuum (black dots), and the difference between exact and 0th DMP approximation (solid red) and exact and 1st DMP approximation (solid magenta) approximations. Bottom panel is similar to middle panel but plotting the fractional differences, $\Delta P / \bar{P}$. The density used is 3.0 g.cm ${ }^{-3}$. 


\section{NOvA}
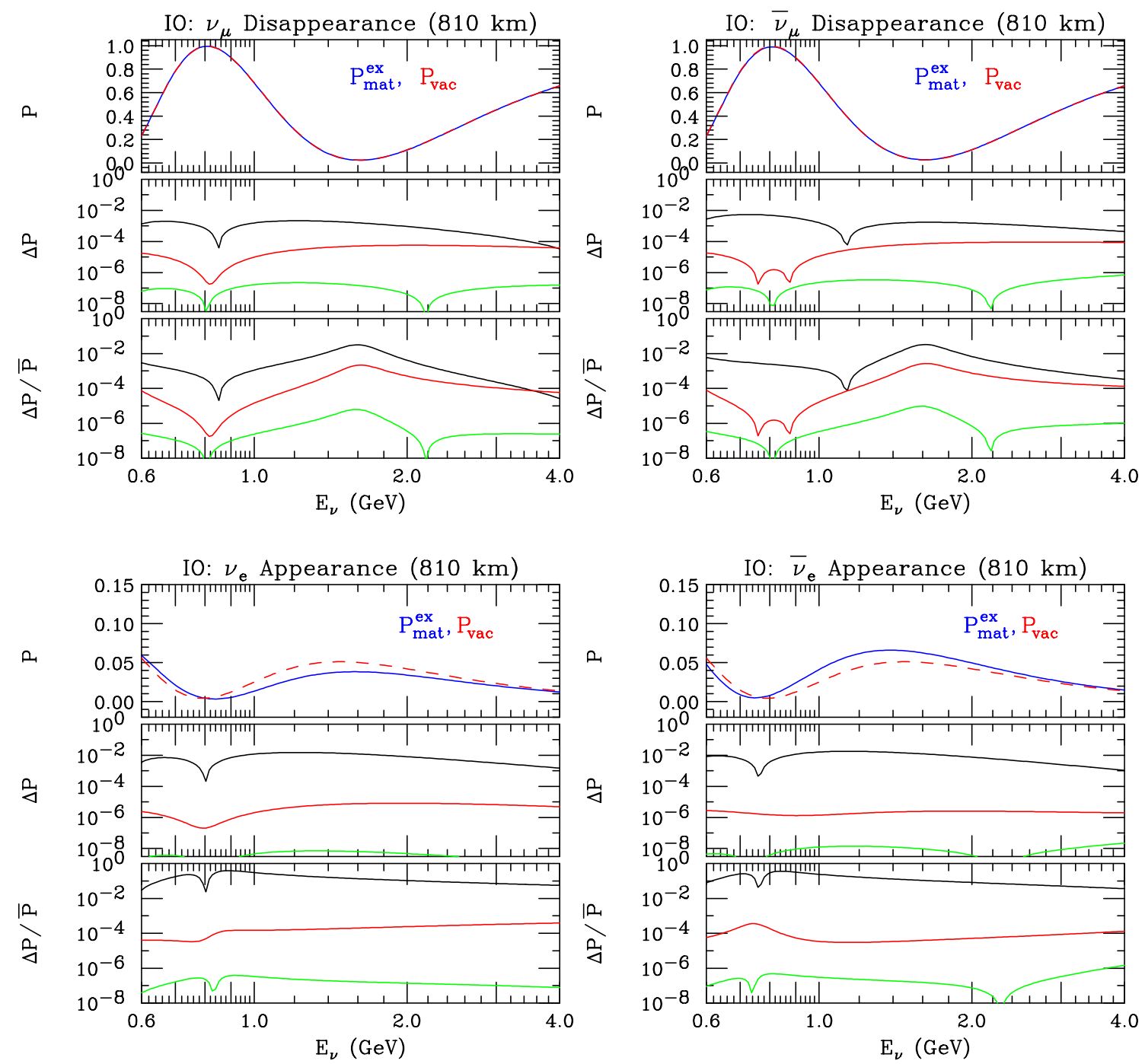

$$
\begin{array}{lll}
\Delta P=\left|P_{\text {mat }}^{e x}-P_{v a c}\right| & \Delta P=\left|P_{\text {mat }}^{e x}-P_{a p p x}^{0 t h}\right| & \Delta P=\left|P_{\text {mat }}^{e x}-P_{\text {app } x}^{1 s t}\right| \\
\bar{P}=\frac{1}{2}\left(P_{m a t}^{e x}+P_{v a c}\right) & \bar{P}=\frac{1}{2}\left(P_{m a t}^{e x}+P_{a p p x}^{0 t h}\right) & \bar{P}=\frac{1}{2}\left(P_{\text {mat }}^{e x}+P_{a p p x}^{1 s t}\right)
\end{array}
$$

Figure 6. NOvA, for inverted ordering (IO): top left figure is $\nu_{\mu}$ disappearance, top right figure is $\bar{\nu}_{\mu}$ disappearance, bottom left figure is $\nu_{\mu} \rightarrow \nu_{e}$ appearance, and bottom right is $\bar{\nu}_{\mu} \rightarrow \bar{\nu}_{e}$ appearance. In each figure, the top panel is exact oscillation probability in matter, $P_{\text {mat }}^{e x}$ (blue dashes) from [11], the zeroth order DMP approximation, $P_{a p p x}^{0 t h}$ (red dashes) from [1] and the vacuum oscillation probability, $P_{v a c}$ (black dots). The Middle panel is difference between exact oscillation probabilities in matter and vacuum (black dots), and the difference between exact and 0th DMP approximation (solid red) and exact and 1st DMP approximation (solid magenta) approximations. Bottom panel is similar to middle panel but plotting the fractional differences, $\Delta P / \bar{P}$. The density used is 3.0 g.cm ${ }^{-3}$. 

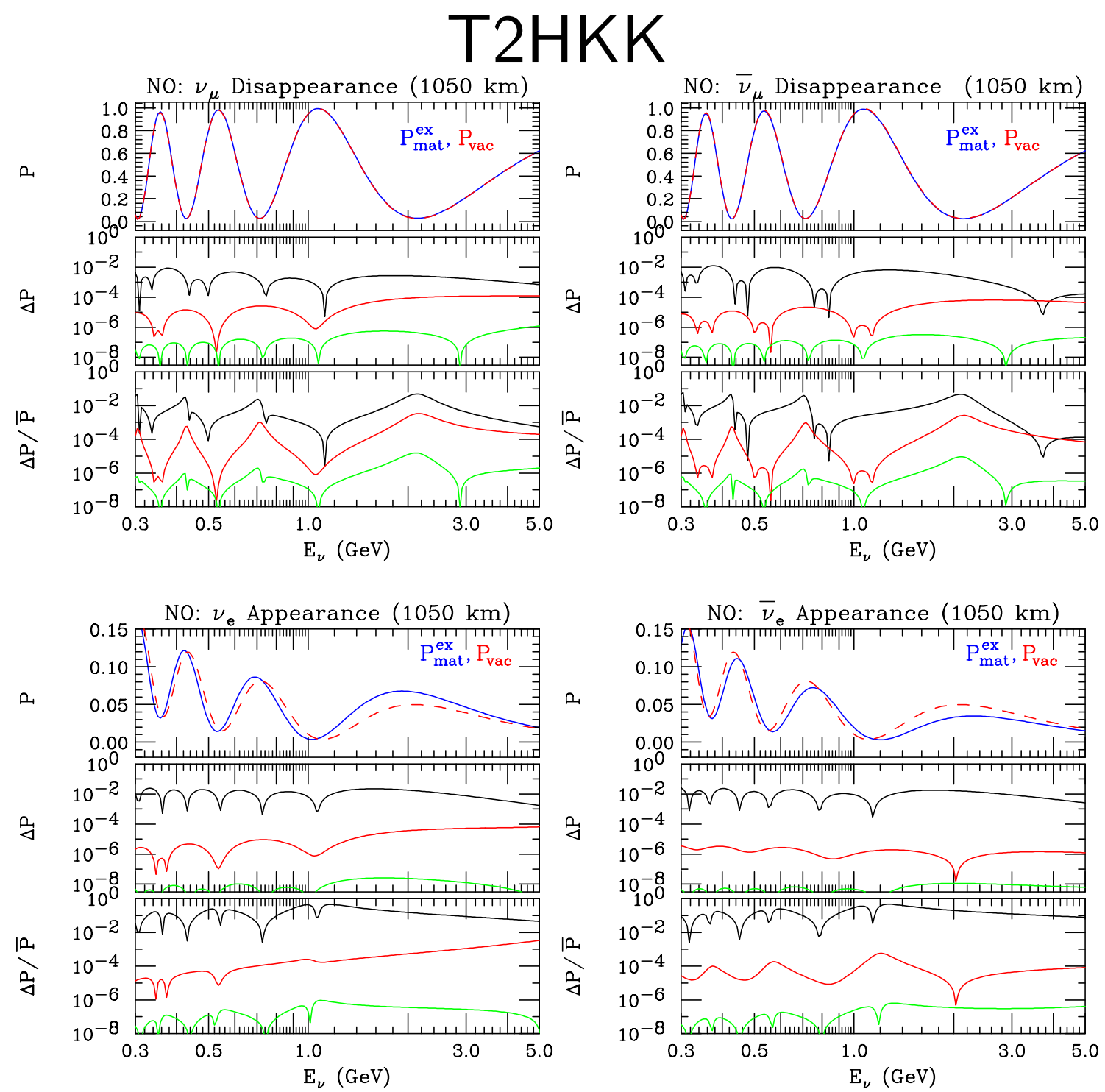

$$
\begin{array}{lll}
\Delta P=\left|P_{m a t}^{e x}-P_{v a c}\right| & \Delta P=\left|P_{m a t}^{e x}-P_{a p p x}^{0 t h}\right| & \Delta P=\left|P_{m a t}^{e x}-P_{a p p x}^{1 s t}\right| \\
\bar{P}=\frac{1}{2}\left(P_{m a t}^{e x}+P_{v a c}\right) & \bar{P}=\frac{1}{2}\left(P_{\text {mat }}^{e x}+P_{a p p x}^{0 t h}\right) & \bar{P}=\frac{1}{2}\left(P_{\text {mat }}^{e x}+P_{a p p x}^{1 s t}\right)
\end{array}
$$

Figure 7. T2HKK, for normal ordering (NO): top left figure is $\nu_{\mu}$ disappearance, top right figure is $\bar{\nu}_{\mu}$ disappearance, bottom left figure is $\nu_{\mu} \rightarrow \nu_{e}$ appearance, and bottom right is $\bar{\nu}_{\mu} \rightarrow \bar{\nu}_{e}$ appearance. In each figure, the top panel is exact oscillation probability in matter, $P_{m a t}^{e x}$ (blue dashes) from [11], the zeroth order DMP approximation, $P_{a p p x}^{0 \text { th }}$ (red dashes) from [1] and the vacuum oscillation probability, $P_{v a c}$ (black dots). The Middle panel is difference between exact oscillation probabilities in matter and vacuum (black dots), and the difference between exact and 0th DMP approximation (solid red) and exact and 1st DMP approximation (solid magenta) approximations. Bottom panel is similar to middle panel but plotting the fractional differences, $\Delta P / \bar{P}$. The density used is $3.0 \mathrm{~g} . \mathrm{cm}^{-3}$. 


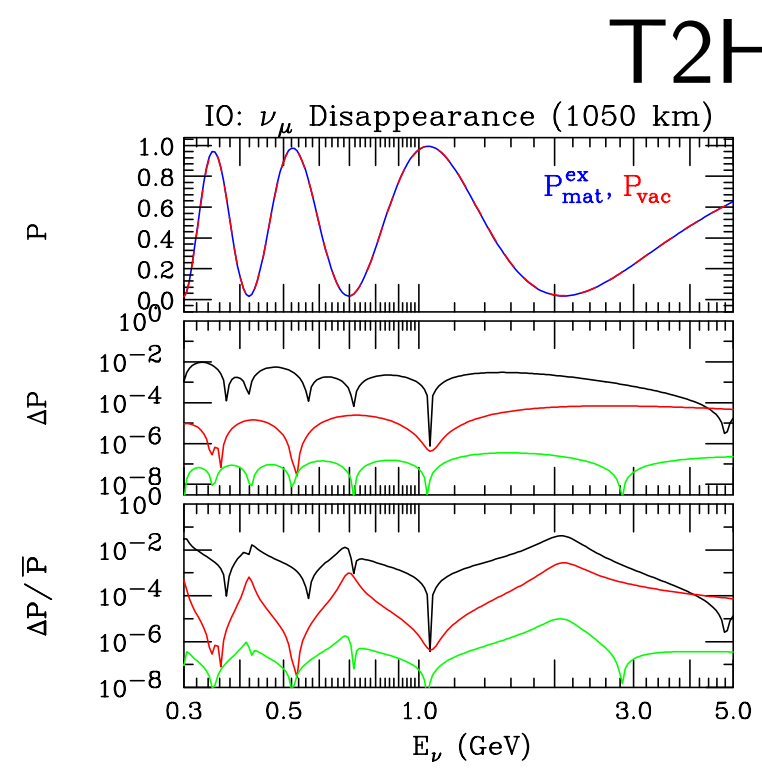

\section{KK}
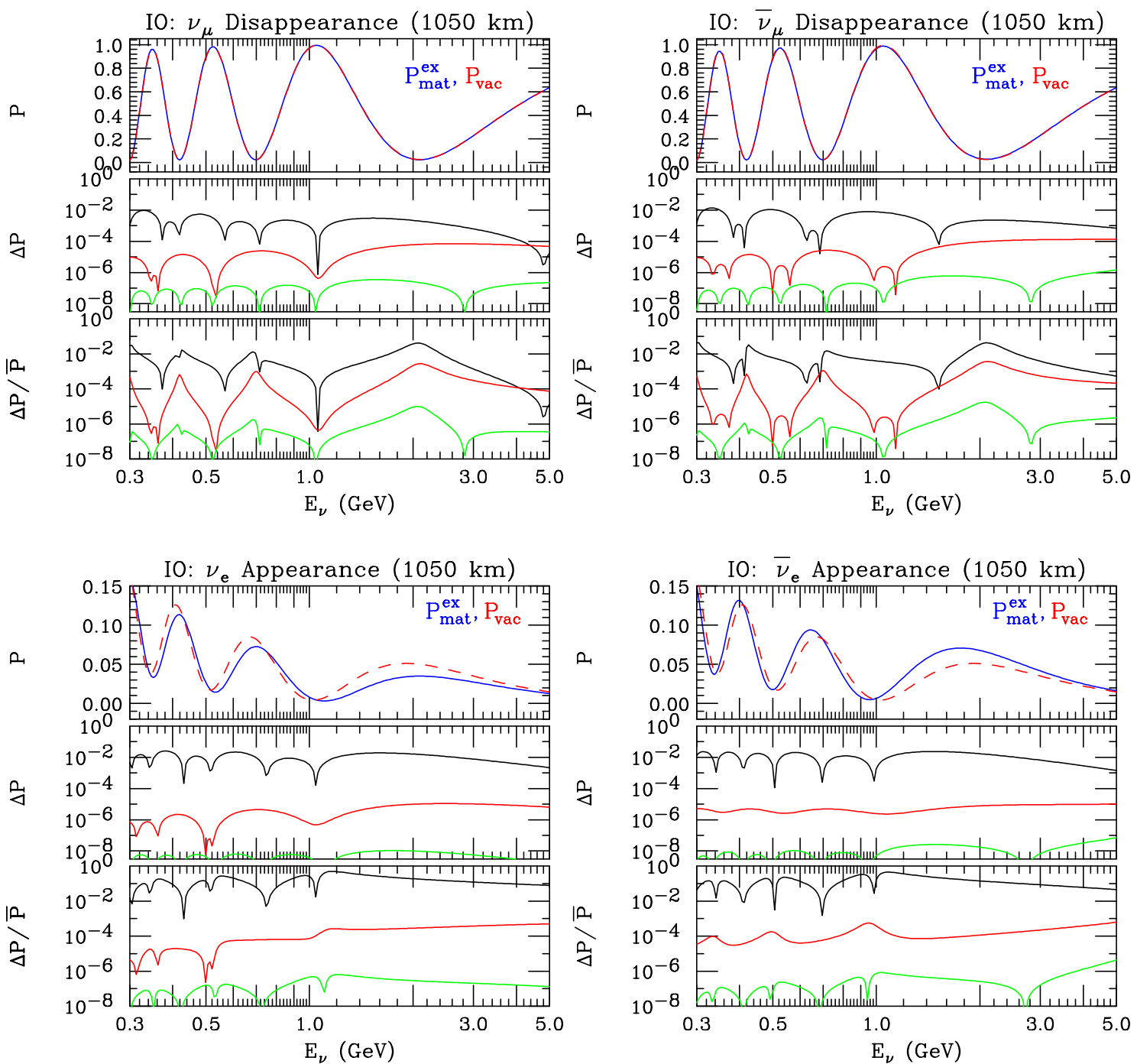

$$
\begin{array}{lll}
\Delta P=\left|P_{m a t}^{e x}-P_{v a c}\right| & \Delta P=\left|P_{\text {mat }}^{e x}-P_{a p p x}^{0 t h}\right| & \Delta P=\left|P_{\text {mat }}^{e x}-P_{a p p x}^{1 s t}\right| \\
\bar{P}=\frac{1}{2}\left(P_{\text {mat }}^{e x}+P_{v a c}\right) & \bar{P}=\frac{1}{2}\left(P_{\text {mat }}^{e x}+P_{a p p x}^{0 t h}\right) & \bar{P}=\frac{1}{2}\left(P_{\text {mat }}^{e x}+P_{a p p x}^{1 s t}\right)
\end{array}
$$

Figure 8. T2HKK, for inverted ordering (IO): top left figure is $\nu_{\mu}$ disappearance, top right figure is $\bar{\nu}_{\mu}$ disappearance, bottom left figure is $\nu_{\mu} \rightarrow \nu_{e}$ appearance, and bottom right is $\bar{\nu}_{\mu} \rightarrow \bar{\nu}_{e}$ appearance. In each figure, the top panel is exact oscillation probability in matter, $P_{m a t}^{e x}$ (blue dashes) from [11], the zeroth order DMP approximation, $P_{a p p x}^{0 \text { th }}$ (red dashes) from [1] and the vacuum oscillation probability, $P_{v a c}$ (black dots). The Middle panel is difference between exact oscillation probabilities in matter and vacuum (black dots), and the difference between exact and 0th DMP approximation (solid red) and exact and 1st DMP approximation (solid magenta) approximations. Bottom panel is similar to middle panel but plotting the fractional differences, $\Delta P / \bar{P}$. The density used is $3.0 \mathrm{~g} . \mathrm{cm}^{-3}$. 

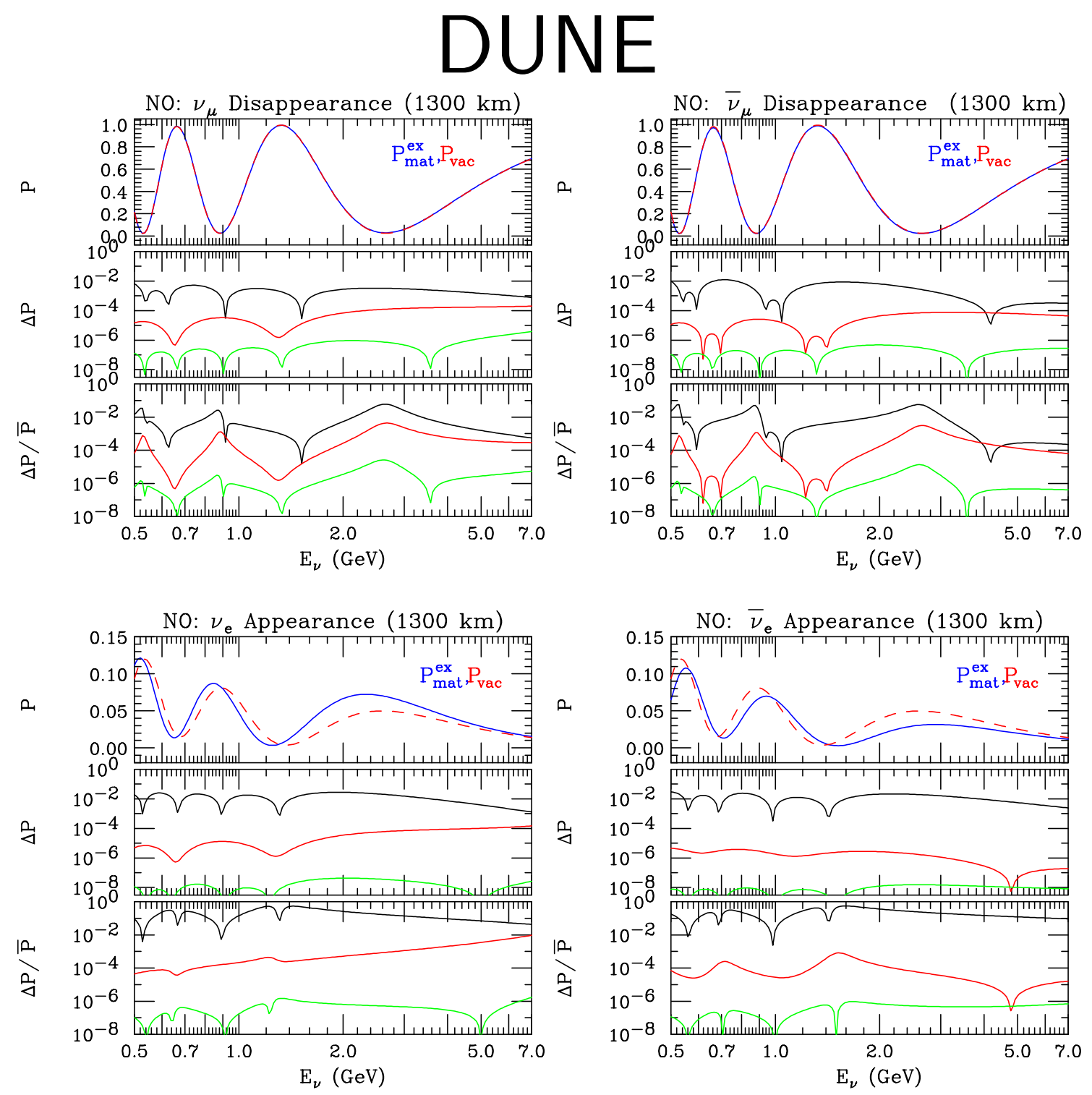

$$
\begin{array}{lll}
\Delta P=\left|P_{\text {mat }}^{e x}-P_{\text {vac }}\right| & \Delta P=\left|P_{\text {mat }}^{e x}-P_{\text {appx }}^{0 t h}\right| & \Delta P=\left|P_{\text {mat }}^{e x}-P_{\text {app }}^{1 s t}\right| \\
\bar{P}=\frac{1}{2}\left(P_{\text {mat }}^{e x}+P_{v a c}\right) & \bar{P}=\frac{1}{2}\left(P_{\text {mat }}^{e x}+P_{a p p x}^{0 t h}\right) & \bar{P}=\frac{1}{2}\left(P_{\text {mat }}^{e x}+P_{\text {app }}^{1 s t}\right)
\end{array}
$$

Figure 9. DUNE, for normal ordering (NO): top left figure is $\nu_{\mu}$ disappearance, top right figure is $\bar{\nu}_{\mu}$ disappearance, bottom left figure is $\nu_{\mu} \rightarrow \nu_{e}$ appearance, and bottom right is $\bar{\nu}_{\mu} \rightarrow \bar{\nu}_{e}$ appearance. In each figure, the top panel is exact oscillation probability in matter, $P_{m a t}^{e x}$ (blue dashes) from [11], the zeroth order DMP approximation, $P_{a p p x}^{0 t h}$ (red dashes) from [1] and the vacuum oscillation probability, $P_{v a c}$ (black dots). The Middle panel is difference between exact oscillation probabilities in matter and vacuum (black dots), and the difference between exact and 0th DMP approximation (solid red) and exact and 1st DMP approximation (solid magenta) approximations. Bottom panel is similar to middle panel but plotting the fractional differences, $\Delta P / \bar{P}$. The density used is 3.0 g.cm $\mathrm{cm}^{-3}$. 


\section{DUNE}
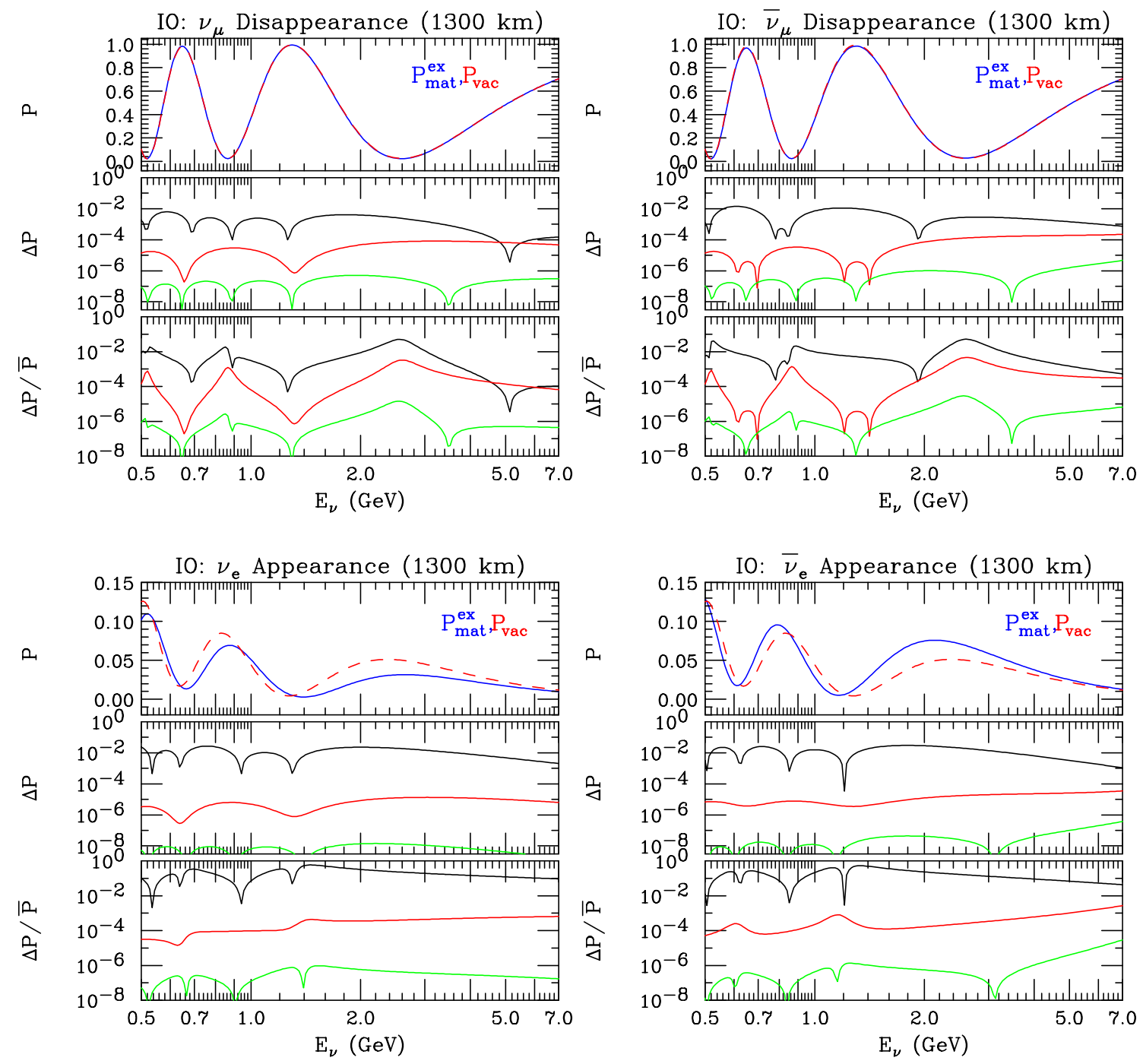

$$
\begin{array}{lll}
\Delta P=\left|P_{m a t}^{e x}-P_{v a c}\right| & \Delta P=\left|P_{\text {mat }}^{e x}-P_{a p p x}^{0 t h}\right| & \Delta P=\left|P_{\text {mat }}^{e x}-P_{\text {appx }}^{1 s t}\right| \\
\bar{P}=\frac{1}{2}\left(P_{\text {mat }}^{e x}+P_{v a c}\right) & \bar{P}=\frac{1}{2}\left(P_{\text {mat }}^{e x}+P_{\text {app }}^{0 t h}\right) & \bar{P}=\frac{1}{2}\left(P_{\text {mat }}^{e x}+P_{\text {app }}^{1 s t}\right)
\end{array}
$$

Figure 10. DUNE, for inverted ordering (IO): top left figure is $\nu_{\mu}$ disappearance, top right figure is $\bar{\nu}_{\mu}$ disappearance, bottom left figure is $\nu_{\mu} \rightarrow \nu_{e}$ appearance, and bottom right is $\bar{\nu}_{\mu} \rightarrow \bar{\nu}_{e}$ appearance. In each figure, the top panel is exact oscillation probability in matter, $P_{m a t}^{e x}$ (blue dashes) from [11], the zeroth order DMP approximation, $P_{a p p x}^{0 t h}$ (red dashes) from [1] and the vacuum oscillation probability, $P_{v a c}$ (black dots). The Middle panel is difference between exact oscillation probabilities in matter and vacuum (black dots), and the difference between exact and 0th DMP approximation (solid red) and exact and 1st DMP approximation (solid magenta) approximations. Bottom panel is similar to middle panel but plotting the fractional differences, $\Delta P / \bar{P}$. The density used is 3.0 g.cm ${ }^{-3}$. 
PBD acknowledges support from the Villum Foundation (Project No. 13164) and the Danish National Research Foundation (DNRF91 and Grant No. 1041811001).

\section{A Asymptotic expansions}

The asymptotic forms of $\widetilde{\Delta m^{2}}$ ee and $\widetilde{\Delta m^{2}} 21$ are given here:

$$
\widetilde{\Delta m^{2}} e= \begin{cases}\Delta m_{e e}^{2}-a \cos 2 \theta_{13}, & |a| \ll\left|\Delta m_{e e}^{2}\right| \\ \Delta m_{e e}^{2}\left|a / \Delta m_{e e}^{2}-\cos 2 \theta_{13}\right|, & |a| \gg\left|\Delta m_{e e}^{2}\right|,\end{cases}
$$

and

$$
\widetilde{\Delta m^{2}}{ }_{21}=\left\{\begin{array}{l}
\Delta m_{21}^{2}-a \cos ^{2} \theta_{13} \cos 2 \theta_{12},|a| \ll \Delta m_{21}^{2} \\
\Delta m_{21}^{2}\left|a^{\prime} / \Delta m_{21}^{2}-\cos 2 \theta_{12}\right|,\left|a^{\prime}\right| \gg \Delta m_{21}^{2}
\end{array}\right.
$$

If $|a| \ll \mid \Delta m_{e e}^{2}$, that is when $E_{\nu} \ll 11\left(\rho / 3 \mathrm{~g} \mathrm{~cm}^{-3}\right) \mathrm{GeV}$, we have,

$$
\begin{aligned}
\sin ^{2} \widetilde{\theta}_{13} & \left.\approx s_{13}^{2}\left[1+2 c_{13}^{2}\left(a / \Delta m_{e e}^{2}\right)+3\left(c_{13}^{2}-s_{13}^{2}\right) c_{13}^{2}\left(a / \Delta m_{e e}^{2}\right)^{2}+\mathcal{O}\left(a / \Delta m_{e e}^{2}\right)^{3}\right)\right] \\
\sin ^{2}\left(\widetilde{\theta}_{13}-\theta_{13}\right) & \approx s_{13}^{2} c_{13}^{2}\left(a / \Delta m_{e e}^{2}\right)^{2}\left[1+2\left(c_{13}^{2}-s_{13}^{2}\right)\left(a / \Delta m_{e e}^{2}\right)+\mathcal{O}\left(a / \Delta m_{e e}^{2}\right)^{2}\right] \\
a^{\prime} & \approx a c_{13}^{2}\left[1-s_{13}^{2}\left(a / \Delta m_{e e}^{2}\right)-s_{13}^{2}\left(c_{13}^{2}-s_{13}^{2}\right)\left(a / \Delta m_{e e}^{2}\right)^{2}+\mathcal{O}\left(a / \Delta m_{e e}^{2}\right)^{3}\right]
\end{aligned}
$$

up to $\mathcal{O}\left(a / \Delta m_{e e}^{2}\right)^{2}$. The expansion for $a^{\prime}$ can be used to calculate $\Delta \widetilde{m^{2}} 31$ as follows,

$$
\Delta{\widetilde{m^{2}}}_{31}= \begin{cases}\Delta m_{31}^{2}+\left(a-a^{\prime}\right)+\frac{1}{2}\left[\Delta \widetilde{m^{2}} 21-\Delta m_{21}^{2}-a^{\prime}\right], & a, a^{\prime}>0 \\ \Delta m_{31}^{2}+\left(a-2 a^{\prime}\right)+\frac{1}{2}\left[\Delta \widetilde{m^{2}}{ }_{21}-\Delta m_{21}^{2}+a^{\prime}\right], & a, a^{\prime}<0\end{cases}
$$

where the quantities in $[\cdots]$ is of $\mathcal{O}\left(\Delta m_{21}^{2}\right)$ for all values of $E_{\nu}$.

As can be seen from figure 1 and figure 2 , both $\Delta \widetilde{m^{2}} 21$ and $\sin ^{2} \widetilde{\theta}_{12}$ make rapid changes in $+150 \mathrm{MeV}$ region. Well away from this region, when $|a| \gg\left|\Delta m_{21}^{2}\right|$, that is $E_{\nu} \gg$ $150\left(\rho / 3 \mathrm{~g} \mathrm{~cm}^{-3}\right) \mathrm{MeV}$, we can write

$$
\Delta \widetilde{m^{2}} 21 \approx\left|a^{\prime}-\Delta m_{21}^{2} \cos 2 \theta_{12}\right|
$$

for $\left|a^{\prime}-\Delta m_{21}^{2} \cos 2 \theta_{12}\right| \gg \Delta m_{21}^{2}$. This can be used to obtain the asymptotic values for neutrino mass squareds in matter, which agree with the values given in DMP.

\section{B Comparison between this addendum and DMP}

In table 2 we give the relationship between the key variables of this Addendum and that of original paper, DMP [1], including the relevant eq. number.

Open Access. This article is distributed under the terms of the Creative Commons Attribution License (CC-BY 4.0), which permits any use, distribution and reproduction in any medium, provided the original author(s) and source are credited. 


\begin{tabular}{|ccc|}
\hline This work & DMP & eq. \# in DMP \\
\hline $\cos 2 \widetilde{\theta}_{13}=$ & $\cos 2 \phi$ & 2.3 .5 \\
$\cos 2 \widetilde{\theta}_{12}=$ & $\cos 2 \psi$ & 2.4 .9 \\
$\Delta \widetilde{m^{2}}$ & $=\Delta \lambda_{+-}$ & 2.3 .3 \\
$\Delta \widetilde{m^{2}}$ & $=\Delta \lambda_{21}$ & 2.4 .5 \\
$\widetilde{m^{2}}$ & $=\Delta \lambda_{31}$ & $2.3 .3+2.4 .5$ \\
\hline
\end{tabular}

Table 2. The connection between the key variables of this paper and those of [1]. Note that the equation numbers match the arXiv version.

\section{References}

[1] P.B. Denton, H. Minakata and S.J. Parke, Compact Perturbative Expressions For Neutrino Oscillations in Matter, JHEP 06 (2016) 051 [arXiv: 1604.08167] [INSPIRE].

[2] H. Nunokawa, S.J. Parke and R. Zukanovich Funchal, Another possible way to determine the neutrino mass hierarchy, Phys. Rev. D 72 (2005) 013009 [hep-ph/0503283] [INSPIRE].

[3] S. Parke, What is $\Delta m_{e e}^{2}$ ?, Phys. Rev. D 93 (2016) 053008 [arXiv: 1601.07464] [InSPIRE].

[4] H. Minakata and S.J. Parke, Simple and Compact Expressions for Neutrino Oscillation Probabilities in Matter, JHEP 01 (2016) 180 [arXiv:1505.01826] [INSPIRE].

[5] H. Minakata, An Effective Two-Flavor Approximation for Neutrino Survival Probabilities in Matter, JHEP 05 (2017) 043 [arXiv: 1702.03332] [INSPIRE].

[6] T2K collaboration, K. Abe et al., The T2K Experiment, Nucl. Instrum. Meth. A 659 (2011) 106 [arXiv: 1106.1238] [INSPIRE].

[7] Hyper-Kamiokande Proto-Collaboration collaboration, K. Abe et al., Physics potential of a long-baseline neutrino oscillation experiment using a J-PARC neutrino beam and Hyper-Kamiokande, PTEP 2015 (2015) 053C02 [arXiv:1502.05199] [INSPIRE].

[8] NOvA collaboration, D.S. Ayres et al., NOvA: Proposal to Build a 30 Kiloton Off-Axis Detector to Study $\nu_{\mu} \rightarrow \nu_{e}$ Oscillations in the NuMI Beamline, hep-ex/0503053 [INSPIRE].

[9] Hyper-Kamiokande Proto- collaboration, K. Abe et al., Physics Potentials with the Second Hyper-Kamiokande Detector in Korea, arXiv:1611.06118 [INSPIRE].

[10] DUNE collaboration, R. Acciarri et al., Long-Baseline Neutrino Facility (LBNF) and Deep Underground Neutrino Experiment (DUNE), arXiv:1512.06148 [INSPIRE].

[11] H.W. Zaglauer and K.H. Schwarzer, The Mixing Angles in Matter for Three Generations of Neutrinos and the MSW Mechanism, Z. Phys. C 40 (1988) 273 [inSPIRE].

[12] S.J. Parke, P.B. Denton and H. Minakata, Analytic Neutrino Oscillation Probabilities in Matter: Revisited, in 2017 International Workshop on Neutrinos from Accelerators (NuFact17) Uppsala University Main Building, Uppsala, Sweden, September 25-30, 2017, 2018, arXiv: 1801.00752 [INSPIRE].

[13] S. Parke and M. Messier, Cross Check of NOvA Oscillation Probabilities, (2018), http://doi.org/10.5281/zenodo.1146747. 